\title{
Detection of Tuberculosis using Hybrid Features from Chest Radiographs
}

\author{
Maham Mehr Awan ${ }^{1}$, Ayesha Fatima², Kinza Mehr Awan ${ }^{3}$ \\ ${ }^{1}$ Department of Software Engineering, University of Central punjab, Lahore,Pakistan \\ ${ }^{2}$ Department of Computer Engineering, National University of Science and Technology, NUST \\ ${ }^{3}$ Department of Software Engineering, University of Management and Technology, UMT \\ 1'mahammehr@ucp.edu.pk,
}

\begin{abstract}
Tuberculosis is a contagious disease, but it's diagnosis is still a difficult and challenging task as it is considered a big threat everywhere on the planet. Literature shows that underdeveloped countries widely use chest radiographs (X ray images) for the diagnosis of tuberculosis. Low accuracy of results and high cost are the two main reasons due to which most of the available methods are not useful for radiologists. In our research, we proposed a detection technique in which features extraction is performed on the basis of their texture, intensity and shape. For evaluating the performance of our proposed methodology, Montgomery Country (MC) dataset is used. It is a publically available data set which consists of $138 \mathrm{CXRs}$; among them, $80 \mathrm{CXRs}$ are normal and $58 \mathrm{CXRs}$ are malignant. The results of the proposed technique have outperformed state of the art methodologies on the MC dataset as it has shown $81.16 \%$ accuracy.
\end{abstract}

Keywords: Tuberculosis (TB), Chest Radiograph (CXR), Classification, Computer-Aided Diagnosis (CAD).

\section{INTRODUCTION}

Tuberculosis is a crucial health issue, on the lungs and causes tuberculosis [2]. especially in underdeveloped countries where poverty and malnutrition reduce resistance to diseases. About one-third of people in the world are suffering from TB [1]. A bacteria, Bacillus Mycobacterium tuberculosis, attacks

Different methods were developed for the diagnosis of TB. Bacilli can be diagnosed from Ziehl-Nelson stained sputum spear images for beaded structures [3]. Detection of tuberculosis from CXR is reliable and fast, especially in less 
developed countries and it has no adverse factor on the human body. A chest radiograph is commonly used to diagnose the conditions that affects lungs. Infiltration, effusion and miliary patterns are typical manifestations of TB [4].

The advent of CXR provided a novel direction to screening/diagnosis using artificial intelligence. Different methods are proposed, but still there is a room for improvements in the research to meet the pragmatic requirements for real-life deployable diagnostic systems. Recently in a survey, it is stated that it took 45 years of analysis, study and research on computer-aided diagnosis (CAD) for chest radiography and different systems are proposed for the diagnosis of tuberculosis. Still there is no perfect system which is able to assist radiologists for decision making [5-7] as cost and accuracy issues are not completely solved. Some methods are too slow and others are not affordable in less developed countries. In [8], lungs are segmented by combined mask with shape and texture descriptors for the histogram to detect TB. Another automated system was proposed to improve the false positive rate by using texture and content based image retrieval features [9]. In [10], TB was detected on the basis of textural, focal and shape abnormality analysis and results of these features are grouped as TB score. As pulmonary detection is the major evaluation of $\mathrm{TB}$ so different methods have been reported in the literature. Chest radiographs are viewed as normal or abnormal on the basis of thoracic edge maps [11]. In [12], localized region-based segmentation and different features were used for TB detection.
The structure of this paper is as follows. Section 2 explains the proposed methodology. Section 3 describes the experimentation, including details of dataset, results and discussion. Section 4 presents the conclusion.

\section{Proposed Methodology}

In the suggested methodology, various features from the input image are extracted and concatenated in the feature vector. For the detection of normal and abnormal images from input images, SVM classifier is used. The flowchart of the recommended method is shown in figure 1.

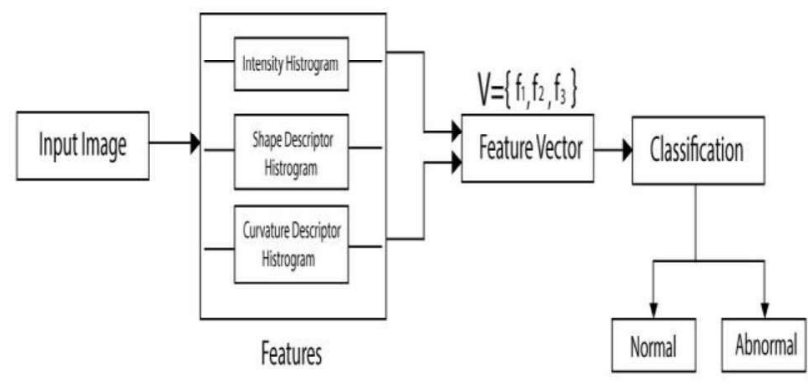

Figure 6: Flowchart of the proposed methodology

\subsection{Feature Extraction}

The key objective of feature extraction is to designate the normal and abnormal patterns of lung regions. Each feature has its own characteristics and details of feature descriptors are described as following.

\subsubsection{Intensity Histogram}

The intensity histogram represents the histogram of intensity pixel values. It represents the number of pixels that have different intensity values of an image [4]. 


\subsubsection{Histogram of Gradient}

In the histogram of gradient (HOG) method, the image is divided into various cells. Each cell has its own histogram based on the pixel values of the cell. The descriptor is computed by combining all histograms of these cells. Good performance of HOG depends upon different parameters i.e. orientation bins and size of descriptor blocks. Parameters of HOG are the following:

- Gamma/Color Normalization: Input pixel can be represented in different ways i.e., grayscale, RGB and LAB color spaces. Color normalization has an uncertain effect because of subsequent descriptor normalization. Color information gives better results over grayscale information.

- Gradient Computation: In HOG, the gradient is computed by Gaussian smoothing and derivative masks. Different smoothing filters and masks are tested to enhance performance. It is observed that an increase in the smoothing scale and an increase in the size of masks that can decrease the performance.

\section{- Spatial/OrientationBinning:}

Spatial/Orientation binning is performed by calculating a weighted vote for an edge positioning histogram channel based on gradient element position on its respective pixel and orientation bins are used to accumulate these votes in cells. As a function of gradient magnitude, the vote is defined, at the pixel of each cell. Gradient magnitude is either itself the magnitude, square of it or square root etc. It is observed that magnitude gives the best result.

\section{- Normalization \& Descriptor Blocks:}

Strength of gradient has variation in brightness and contrast of foreground and background, so effective normalization of contrast is important for high performance and good efficiency. Various normalization techniques are assessed and evaluated, mostly based upon grouping up cells to form larger blocks and normalize contrast of every cell separately. HOG is used in many detection systems [13].

\subsubsection{Gradient Image Histogram}

Gradient magnitude histogram is used to represent the statistics of an image, in which the magnitude of an image plane maps the image plane. It used to determine the directional change in an image. [14].

\subsubsection{Curvature Descriptor Histogram}

Curvature descriptor histogram is based on the curvature of an object and eigenvalues as shown in equation 1 , and are calculated from Hessian matrix:

$$
C D=\tan ^{-1}\left(\frac{\sqrt{\lambda_{1}^{2}+\lambda_{2}^{2}}}{1+I(a, b)}\right)(1)
$$

where $0 \leq \mathrm{CD} \leq \pi / 2$ and $\mathrm{I}(\mathrm{a}, \mathrm{b})$ represents the pixel value of an image. Normalization is done w.r.t intensity due to which this descriptor is independent of the brightness of the image. 


\subsubsection{Shape Descriptor Histogram}

The shape descriptor histogram is based on the shape and geometry of an image and it is calculated by equation 2 .

$$
S D=\tan ^{-1}\left(\frac{\lambda_{1}}{\lambda_{2}}\right)(2)
$$

with $\lambda_{1} \leq \lambda_{2}$ where $\lambda_{1}$ and $\lambda 2$ represent the Hessian matrix's eigenvalues. Eigenvalues of a Hessian matrix are computed to determine the local behavior of an image using a multiscale approach by Frangi et al.[15]. It is considered a filtering process that extracts geometric structure from an image that can be in the form of tabular. The behavior of an image I, can be analyzed by Taylor expansion in the neighborhood of a point a0 as follows: -

$I(a, s) \approx I\left(a_{0}, s\right)+\left(x-a_{0}\right)^{T} \nabla I\left(a_{0}, s\right)+\frac{1}{2}\left(x-a_{0}\right)^{T} H\left(a_{0}, s\right)\left(a-a_{0}\right)$

Where $\nabla I\left(a_{0}, s\right)$ represents gradient vector and $H\left(a_{0}, s\right)$ represents the Hessian matrix of an image calculated at scale $s$. Scale $s$ is calculated by the convolution of derivatives of Gaussian according to scale-space theory. It is shown as:

$$
\frac{\partial}{\partial x} I\left(a_{0}, s\right)=s^{\gamma} I\left(a_{0}\right) * \frac{\partial}{\partial x} G\left(a_{0}, s\right)(4)
$$

Where the d-dimension Gaussian for pixel $\mathrm{a}_{0}$ and scale $\mathrm{s}$.

$$
G\left(x_{0}, s\right)=\frac{1}{\sqrt{\left(2 \pi s^{2}\right)^{d}}} e^{-\frac{\left\|x_{0}\right\|^{2}}{2 s^{2}}}
$$

Frangi filters are used to improve blood vessels, which have thin and tiny elongated shapes, but our goal is to detect lung nodules which can be detected by extracting spherical or elliptical shapes. Therefore structure response SR filter is used based on $\lambda_{1}$ and $\lambda_{2}$ as follows:

$$
S R=1-e^{-\sqrt{k \cdot\left|\lambda_{1} * \lambda_{2}\right|}}
$$

The eigenvalues used for shape and curvature descriptors are those who give the best filter results over different Gaussian filters namely $\mathrm{s}=2,4,6,8 \ldots .20$. All descriptors except area are quantized into 32 histogram bins, so total features are $\left(5^{*} 32\right)=160$ in number. All features are concatenated in the form of the feature vector and given to classifiers for the detection of tuberculosis.

\subsubsection{Classification}

Classification s used to detect the abnormality in chest radiographs. Support vector machine SVM classifier is used in our experiments. In our proposed methodology, different extracted features are concatenated in the form of a feature vector and given to the classifier. The SVM is trained by calculating the features vectors of data of known labels [16-17]. SVM classifies the chest radiographs as normal and abnormal according to distance from the hyperplane.

\section{EXPERIMENTATION}

\subsection{Dataset}

MC dataset is a demonstrative subset of big Xrays respiratory system, which is collected over a number of years underneath the Montgomery Country's program of tuberculosis control [8]. The dataset consists of 138 CXRs; among them, normal X-rays are 80 and remaining abnormal $\mathrm{X}$-rays are 58 having different manifestations of TB. All the images have 12-bit grayscale; abnormal CXRS contains various appearances of TB, including effusion and miliary patterns. 


\subsection{Results and Discussion}

Efficiency and performance evaluation of anticipated methodology is done on MC dataset. Various features are extracted from an image and performance is evaluated on different combinations of features. Leave-one-out validation scheme is used with SVM classifier. In leave-one-out scheme, classify each feature vector with a classifier trained on a lingering feature vector. Evaluation measures include the accuracy, specificity and sensitivity of a system. Specificity calculates the proportion of normal correctly classified radiographs, also known as true negative' rate. Sensitivity measures the ratio of abnormal correctly identified chest radiographs also called true positive rate. The classifier correctly scored some radiographs as normal and abnormal, as shown in figure 2.
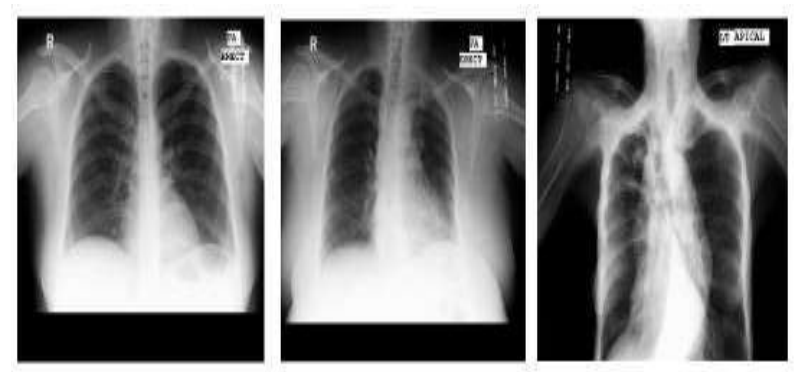

Figure 2: correctly classified images a) classifier scored as normal b) classifier scored as abnormal c) classifier scored as abnormal CXR

Table 3. performance of descriptors individually using proposed method

\begin{tabular}{|c|c|c|c|}
\hline $\begin{array}{c}\text { Feature } \\
\mathrm{s}\end{array}$ & $\begin{array}{c}\text { Accurac } \\
\mathrm{y}\end{array}$ & $\begin{array}{c}\text { Specificit } \\
\mathrm{y}\end{array}$ & $\begin{array}{c}\text { Sensitivit } \\
\mathrm{y}\end{array}$ \\
\hline $\mathrm{IH}$ & 0.5580 & 0.6111 & 0.4583 \\
\hline $\mathrm{GM}$ & 0.5942 & 0.6933 & 0.4762 \\
\hline $\mathrm{SD}$ & 0.6739 & 0.7045 & 0.6200 \\
\hline $\mathrm{CD}$ & 0.5725 & 0.5926 & 0.5439 \\
\hline HOG & 0.6449 & 0.5667 & 0.7917 \\
\hline
\end{tabular}

Table 4. Performance of the proposed method using hybrid features

\begin{tabular}{|c|c|c|c|}
\hline Features & $\begin{array}{c}\text { Accurac } \\
\mathbf{y}\end{array}$ & $\begin{array}{c}\text { Specificit } \\
\mathbf{y}\end{array}$ & $\begin{array}{c}\text { Sensitivit } \\
\mathrm{y}\end{array}$ \\
\hline $\mathrm{SD}+\mathrm{IH}$ & 0.7826 & 0.8276 & 0.7059 \\
\hline $\mathrm{SD}+\mathrm{CD}$ & 0.7754 & 0.8140 & 0.7115 \\
\hline $\begin{array}{c}\text { SD+CD } \\
+ \text { IH }\end{array}$ & $\mathbf{0 . 8 1 1 6}$ & $\mathbf{0 . 8 2 7 6}$ & $\mathbf{0 . 7 8 4 3}$ \\
\hline $\begin{array}{c}\text { SD } \\
+\mathrm{CD}+\mathrm{G} \\
\mathrm{M}\end{array}$ & 0.6304 & 0.6761 & 0.5821 \\
\hline $\begin{array}{c}\text { SD+HO } \\
\mathrm{G}\end{array}$ & 0.6159 & 0.6818 & 0.5000 \\
\hline \multicolumn{2}{|c|}{} & & \\
\hline
\end{tabular}

Table 1 and Table 2 show the accuracy, specificity and sensitivity of individual and hybrid features using the proposed methodology. Classification is performed on individual features as well as different combinations of features. The high accuracy of $67.39 \%$ is achieved among individuals with shape descriptor histograms having specificity and sensitivity of $70.45 \%$ and $60 \%$, respectively. The high accuracy of $81.16 \%$ is achieved among hybrid features with a combination of shape, curvature and intensity histogram descriptor having specificity and sensitivity of $82.76 \%$ and $78.43 \%$, respectively. It concludes from Table II that the performance of classifiers can decrease by increasing features so only three descriptors are given to classifier, which gives best results. The confusion matrix of hybrid features that give highest accuracy with $81.16 \%$ is shown in Table 3. Our methodology gives best result with a combination of shape, curvature and intensity histogram descriptors. 
Table 5. confusion matrix of the proposed methodology

\begin{tabular}{|l|l|l|}
\hline & Normal & Abnormal \\
\hline Normal & 72 & 15 \\
\hline Abnormal & 11 & 40 \\
\hline
\end{tabular}

Table 6. performance comparison of proposed methodology and state

\begin{tabular}{|l|l|l|}
\hline \multicolumn{1}{|c|}{ Author } & \multicolumn{1}{|c|}{ Year } & Accuracy \\
\hline $\begin{array}{l}\text { Jaeger et } \\
\text { al.[8] }\end{array}$ & 2012 & $75 \%$ \\
\hline $\begin{array}{l}\text { Jaeger et } \\
\text { al.[9] }\end{array}$ & 2014 & $78.3 \%$ \\
\hline $\begin{array}{l}\text { Santosh et } \\
\text { al.[11] }\end{array}$ & 2015 & $78.57 \%$ \\
\hline $\begin{array}{l}\text { Proposed } \\
\text { Method }\end{array}$ & 2016 & $81.16 \%$ \\
\hline
\end{tabular}

Different CAD system has been developed to detect tuberculosis. Table 4 shows the comparison of different techniques in which the highest accuracy of $78.5 \%$ is measured. So, the proposed system clearly exceeds the present state of the art techniques applied to the MC dataset.

\section{CONCLUSION}

In this paper, a system is proposed in which various features are extracted and different combinations of features are given to classifier, but the combination of intensity histogram, curvature and shape descriptor histogram gives the best result. Performance is evaluated on standard publically available MC dataset, which contains 80 normal chest radiographs and 58 abnormal radiographs with a different manifestation of tuberculosis. SVM classifier is used and achieves an accuracy of $81.16 \%$. In the future, we can improve the performance by applying segmentation to extract the region of interest before feature extraction.

\section{References}

[1] World Health Organization. (2010). World health statistics 2010. World Health Organization.

[2] World Health Organization. (2011). The global plan to stop TB 2011-2015: transforming the fight towards elimination of tuberculosis.

[3] World Health Organization. (2012). The global plan to stop TB 2011-2015: transforming the fight towards elimination of tuberculosis.

[4] Folio, L. R. (2012). Chest imaging: an algorithmic approach to learning. Springer Science \& Business Media.

[5] Van Ginneken, Bram, Laurens Hogeweg, and Mathias Prokop. "Computer-aided diagnosis in chest radiography: Beyond nodules." European Journal of Radiology 72.2 (2009): 226-230.

[6] Lodwick, Gwilym S. "Computer-aided Diagnosis in Radiology: A Research Plan." Investigative Radiology 1.1 (1966): 72-80. 
[7] Lodwick, Gwilym S. "Computer-aided Diagnosis in Radiology: A Research Plan." Investigative Radiology 1.1 (1966): 72-80.

[8] Jaeger, Stefan, et al. "Detecting tuberculosis in radiographs using combined lung masks." $2012 \quad$ Annual InternationalConference of the IEEE Engineering in Medicine and Biology Society. IEEE, 2012.

[9] Jaeger, Stefan, et al. "Automatic tuberculosis screening using chest radiographs." IEEE transactions on medical imaging 33.2 (2014): 233-245.

[10] Hogeweg, Laurens, et al. "Automatic detection of tuberculosis in chest radiographs using a combination of textural, focal, and shape abnormality analysis." IEEE transactions on medical imaging 34.12 (2015): 2429-2442.

[11] Santosh, K. C., et al. "Automatic Pulmonary Abnormality Screening Using Thoracic Edge Map." 2015 IEEE 28th International Symposium on ComputerBased Medical Systems. IEEE, 2015.

[12] Das, Hrudya, and Ajay Nath. "An Efficient Detection of Tuberculosis from Chest Xrays." International Journal 3.5 (2015).
[13] Dalal, Navneet, and Bill Triggs. "Histograms of oriented gradients for human detection." 2005 IEEE Computer Society Conference on Computer Vision and Pattern Recognition (CVPR'05). Vol. 1. IEEE, 2005.

[14] Maccato, A. "The image gradient histogram and associated orientation signatures." Circuits and Systems, 1995. ISCAS'95., 1995 IEEE International Symposium on. Vol. 1. IEEE.

[15] Frangi, Alejandro F., et al. "Multiscale vessel enhancement filtering." International Conference on Medical Image Computing and Computer-Assisted Intervention. Springer Berlin Heidelberg, 1998.

[16] Vapnik, Vladimir. The nature of statistical learning theory. Springer Science \& business media, 2013.

[17] Burges, Christopher JC, Bernhard Scholkopf, and Alexander J. Smola, eds. Advances in kernel methods: support vector learning. Cambridge, MA, USA:: MIT press, 1999. 